\title{
CARACTERÍSTICAS DE LOS TERCEROS MOLARES INFERIORES IMPACTADOS OBSERVADOS POR MEDIOS RADIOGRÁFICOS. UNIVERSIDAD CATÓLICA DE SANTIAGO DE GUAYAQUIL, 2014
}

IMPACTED LOWER THIRD MOLARS, CHARACTERISTICS OBSERVED BY RADIOGRAPHIC MEANS. UNIVERSIDAD CATÓLICA DE SANTIAGO DE GUAYAQUIL (UCSG), 2014

CARACTERISTICAS DOS TERCEIROS MOLARES INFERIORES IMPACTADOS OBSERVADAS POR MEIOS RADIOGRÁFICOS. UNIVERSIDADE CATÓLICA

DE SANTIAGO DE GUAYAQUIL, 2014

\author{
PAMELA MOREIRA ZEVALLOS', JORGE BARONA TERÁN', JHONNY MOREIRA BOLAÑOS ${ }^{2}$ \\ 1 Universidad Católica de Santiago de Guayaquil; Guayaquil, Ecuador \\ 2 Universidad de Guayaquil, Ecuador
}

RESUMEN

Objetivo: establecer por medio de un estudio radiográfico las características de los terceros molares inferiores impactados con respecto a la posición, la morfología, números de las raíces y la relación con el conducto mandibular. Diseño: se realizó un diseño analítico descriptivo transversal desde el 15 noviembre al 21 diciembre del 2014 en la clínica de odontología de la UCSG, donde se evaluaron historias clínicas y radiografías de 119 pacientes que acudieron para el procedimiento de extracción de terceros molares. Resultados: se estableció que la mayor frecuencia de extracciones que se realizan son las del sexo femenino en las edades de 21- 25 años, y además prefieren extraerse ambos terceros molares inferiores en una misma cita. Se demostró también que la posición según la clasificación de Winter, fue la meso-angulada en ambos lados y según Pell y Gregory fue All, los cuales tenían en mayor frecuencia dos raíces (birradiculares) y la posición en relación al conducto es la tipo II con indicador radiológico tipo I. Conclusión: hacer énfasis en el diagnóstico radiográfico antes de realizar una intervención quirúrgica de extracción de terceros molares infeirores, para así estar preparados para complicaciones que puedan ocurrir en la clínica de Odontología-UCSG.

PALABRAS CLAVE: posición, morfología, dientes impactados, dientes molares.

ABSTRACT

Objective: establish through a radiographic study the characteristics of impacted lower third molars, concerning the position, morphology, root numbers, and the relationship with the mandibular canal. Design: a cross-sectional descriptive-analytical design was carried out from november 15 to december 21, 2014, at the UCSG dentistry clinic, where the medical records and radiographs of 119 patients who attended for the third molar extraction procedure were evaluated. Results: it was established the highest frequency of extractions performed are those of the female sex between the ages of 21 and 25 , and they also prefer to extract both lower third molars in the same appointment. Was also shown that the position according to Winter's classification was meso-angulated on both sides and according to Pell and Gregory, were more frequently All, which had two roots (bi radicular), additionally, the position about the canal is type II with type I radiological indicator. Conclusion: to emphasize the radiographic diagnosis before performing a surgical intervention of extraction by third lower molars, with the purpose to be prepared for complications that may occur in the Dentistry-UCSG clinic.

KEYWORDS: position, morphology, impacted teeth, molar teeth.

RESUMO

Objetivo: estabelecer por meio de estudo radiográfico as características dos terceiros molares inferiores impactados quanto à posição, morfologia, número de raízes e relação com o canal mandibular. Delineamento: delineamento analítico descritivo transversal foi realizado de 15 de novembro a 21 de dezembro de 2014 na clínica odontológica da UCSG, onde foram avaliados prontuários e radiografias de 119 pacientes que compareceram ao procedimento de exodontia do terceiro molar. Resultados: constatou-se que a maior frequência de extrações realizadas são as do sexo feminino entre 21 a 25 anos, e também preferem extrair os dois terceiros molares inferiores na mesma consulta. Também foi demonstrado que a posição segundo a classificação de Winter era mesoangulada em ambos os lados e segundo Pell e Gregory era All, que tinha duas raízes (birradiculares) com mais frequência e a posição em relação ao canal é do tipo II. com indicador radiológico tipo I. Conclusão: enfatizar o diagnóstico radiográfico antes de realizar uma intervenção cirúrgica de exodontia de terceiros molares inferiores, a fim de estar preparado para complicações que possam ocorrer na Clínica Odontológica-UCSG PALAVRAS-CHAVE: posição, morfologia, dentes impactados, dentes molares. 


\section{INTRODUCCIÓN}

Los terceros molares son las únicas piezas que culminan su crecimiento después de la pubertad y los cuales tienen un periodo de desarrollo que puede durar más de los 10 años. La edad normal de erupción del tercer molar inferior es desde los 16 a los 25 años, las ubicaciones de dichas piezas pueden cambiar en el transcurso del periodo de erupción. Los terceros molares son las piezas que casi siempre encontramos dentro del hueso del maxilar inferior, ya que son los últimos en erupcionar y en muchos casos el tamaño de la mandíbula es pequeña en ancho y longitud comparado al ancho mesiodistal y vestíbulo lingual de los terceros molares inferiores. Por lo tanto, esta mala posición que se origina al momento de la erupción, puede provocar múltiples complicaciones como por ejemplo, quistes dentigeros, problemas de articulación temporomandibular, pericoronaritis, lesiones tumorales, etc. ${ }^{1-6}$

La morfología oclusal muy cambiante ya que puede presentarse desde 5 a más cúspides, con una corona más grande que la del segundo molar inferior. En cambio en la morfología radicular observamos que podemos encontrar una o varias raíces, en general los terceros molares superiores presentan 3 raíces mientras que en los terceros molares inferiores dos. Como previamente se ha mencionado, la desigualdad que hay entre el espacio disponible alveolar a nivel mandibular con la sumatoria de las dimensiones de los dientes lo que da como resultado un espacio insuficiente, lo que provoca que los terceros molares presenten dificultades al momento de terminar su erupción y así de esta manera desarrollándose a nivel interóseo. ${ }^{7-11}$

La impactación sucede cuando una pieza dental no termina su crecimiento completamente, este estará total o parcialmente retenido y la cual se encuentra en el hueso sin poder erupcionar en su totalidad, en el rango de tiempo normal con respecto a la edad del paciente. Este no ha perforado la mucosa y por ende no ha llegado a una posición normal dentro de la arcada dentaria. La clasificación de Winter está basada en la inclinación del eje mayor del tercer molar con relación al segundo molar, por lo cual encontramos siete variaciones: vertical, mesioangular, distoangulado, horizontal, vestibular, lingual, transversal. ${ }^{12-15}$

En el gráfico 1, se realizó estudio de un universo de 100 pacientes, se realizaron 50 muestras de técnicas de anesthesia de tipo Gow Gates y 50 muestras de anesthesia de tipo Akinosi.
A

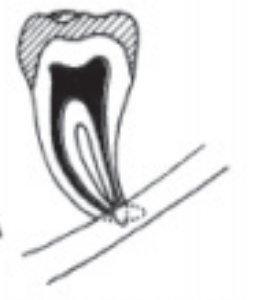

OSCURECTMENTO RADICULAR APICAL.

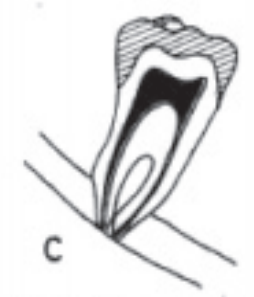

ESTGECHAMENTO RAOKU.AR APICAL

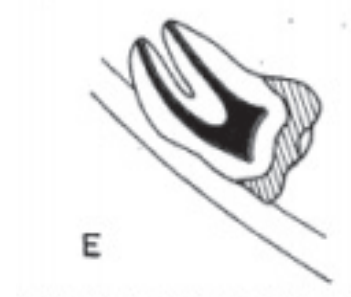

INTERRUPCION DE LA CORTICAL DEL CONDUCTO.
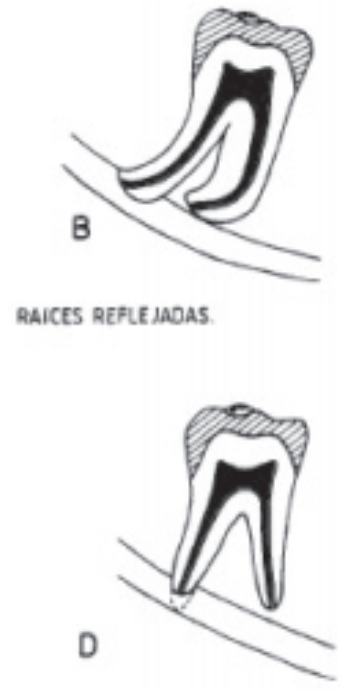

AARCES COSCURAS Y BIFIDAS
RAICES REFLE JADAS

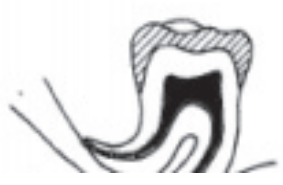

Figura 1. Indicadores radiológicos de lesión nerviosa. Fuente: Virgiti A, criterios de extracción de terceros molares incluidos.

\section{METODOLOGÍA}

Se realizó un estudio analítico descriptivo de tipo transversal en la clínica de odontología UCSC de 119 pacientes teniendo como criterio de inclusión que presenten terceros molares inferiores y que los pacientes tenga la edad de más 17 años, y con criterio de exclusión cuando dichos pacientes solo presenten molares superiores, que no presenten molares inferiores y que el paciente tenga menos de 17 años, dichos pacientes registrados para el procedimiento de extracción de terceros molares en el periodo comprendido desde el 15 de noviembre al 21 de diciembre del 2014. Las exodoncias eran realizadas por estudiantes de los últimos ciclos de las carreras los cuales contaban con las radiografías pertinentes. ${ }^{16,17}$. 
Se consideraron como variables de estudio la edad, el género, la ubicación, clasificación de Winter, Clasificación de Pell y Gregory, morfología radicular y relación del tercer molar con el conducto mandibular dependiendo del tipo de posición se precedía a analizar con el indicador radiológico. ${ }^{18,19}$

La clasificación de Winter está basada en la inclinación del eje mayor del tercer molar con relación al segundo molar, por lo cual encontramos siete variaciones: ${ }^{20,21}$

- Vertical: (El eje mayor del diente retenido y el eje longitudinal del segundo molar se encuentran paralelos. ${ }^{20,21}$ )

- Mesioangular: (El tercer molar retenido está inclinado hacia al segundo molar en dirección mesial. ${ }^{20,21}$ )

- Distoangulado (El eje mayor del tercer molar retenido está inclinado hacia distal del segundo molar. $\left.{ }^{20,21}\right)$

- Horizontal: (Se dirige la superficie oclusal en dirección a la corona y raíz del segundo molar. ${ }^{20,21)}$

- Vestibular: (Dirección hacia vestibular. ${ }^{20,21)}$.

- Lingual: (Se encuentra en dirección hacia lingual. ${ }^{20,21)}$

- Transversal: (Posición horizontal dirigiéndose en un sentido vestibulolingual. 20,21 )

Pell y Gregory - Relación con el segundo molar y la borde anterior de la rama ascendente mandibular. ${ }^{20,21}$

- Clase I: cuando es mayor la dimensión de la corona en sentido mesiodistal comparado con el espacio que encontramos entre el segundo molar y la borde anterior de la rama ascendente mandibular. ${ }^{20,21}$

- Clase II: parte del hueso recubre la porción distal de la corona de tercer molar retenido. ${ }^{20,21}$

- Clase III: se encuentra inmerso en el borde anterior de rama ascendente mandibular. ${ }^{20,21}$

Pell y Gregory - Profundidad del Tercer Molar en el Hueso Mandibular. 22,23

- Posición A: El punto más alto del tercer molar está a la altura o más arriba de la superficie oclusal. 22,23

- Posición B: El punto más alto del tercer molar se encuentra entre el plano oclusal y la línea cervical del segundo molar. ${ }^{22,23}$
- Posición C: El punto más alto del tercer molar está a la altura o por debajo de la línea cervical el segundo molar. ${ }^{22,23}$

La imagen radiográfica del conducto dentario es una banda con mayor radiolucidez en comparación con las trabéculas óseas, se observa como dos líneas paralelas de mayor radiopacidad. El conducto dentario va desde la espina de Spix finalizando en los agujeros mentonianos. En cuanto a las posibles ubicaciones se definieron 7 indicadores radiológicos de la lesión nerviosa y son: oscurecimiento radicular apical,raíces, reflejadas, estrechamiento radicular apical, raíces oscuras y bífidas, interrupción de la cortical del conducto, desviación del conducto dentario inferior, estrechamiento del conducto dentario 22,23

Para el análisis de los resultados se procedió a realizar consentimiento informado, una vez aceptado se continuó a llenar la hoja de registro con datos radiológicos. Para el análisis descriptivo con porcentaje, fueron utilizadas las medidas de resumen y para las variables cualitativas la distribución porcentual con la ayuda del programa Microsoft Excel 2010 Office de Windows. El trabajo de investigación sigue las recomendaciones de la Declaración de Helsinki, (Figuras $1-3) .{ }^{22,23}$

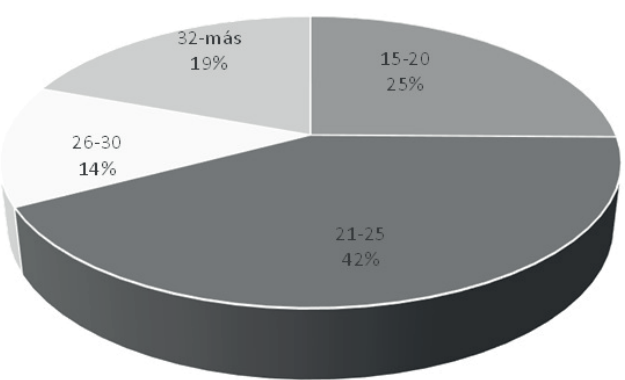

Figura 1. Distribución porcentual de universo y muestra por edad. Fuente: pacientes de la Clínica de Odontología, Universidad Católica de Santiago de Guayaquil.

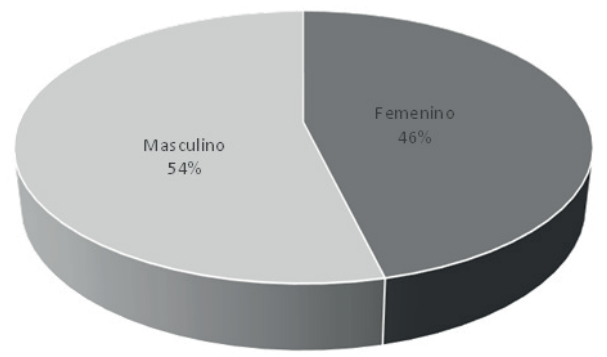

Figura 2. Distribución porcentual de universo y muestra por género. Fuente: pacientes de la Clínica de Odontología, Universidad Católica de Santiago de Guayaquil. 


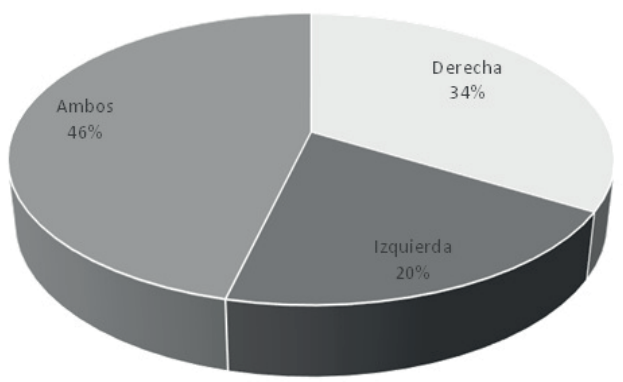

Figura 3. Distribución porcentual de universo y muestra por ubicación. Fuente: pacientes de la Clínica de Odontología, Universidad Católica de Santiago de Guayaquil.

RESULTADOS

Se establece que la extracción de terceros molares inferiores retenidos presenta mayor prevalencia en el grupo de edades entre 21 - 25 en el sexo femenino, los que preferían realizarse la extracción de ambos en el mismo día. Con respecto al eje longitudinal del segundo molar que aparece con mayor frecuencia es la meso-angulado, tanto en el lado derecho como en el izquierdo. Se concluye que la posición con respecto al borde anterior de rama ascendente según Pell y Gregory que aparece con mayor frecuencia en el lado derecho como en el izquierdo, es el tipo II y con respecto a la profundidad es el tipo $\mathrm{A}$ en ambos lados.

En la morfología radicular predominó la birradicular en el lado derecho y en el izquierdo. La posición del canal mandibular según Carter y Ken, en el lado derecho como en el izquierdo es el tipo II. La posición del canal mandibular según Carter y Ken en el tipo I, el indicador radiológico con más frecuencia fue en el lado derecho el oscurecimiento radicular, mientras que en el lado izquierdo el estrechamiento radicular.

TABLA 1. DISTRIBUCIÓN PORCENTUAL DE UNIVERSO Y MUESTRA DE LAS POSICIONES MÁS FRECUENTES CON RESPECTO A LA PROFUNDIDAD SEGÚN PELL Y GREGORY EN PACIENTES QUE FUERON ATENDIDOS POR EXTRACCIÓN DE TERCEROS MOLARES INFERIORES RETENIDOS. FUENTE: PACIENTES DE LA CLÍNICA DE ODONTOLOGÍA - UNIVERSIDAD CATÓLICA DE SANTIAGO DE GUAYAQUIL PROFUNDIDAD RELATIVA SEGÚN PELL Y GREGORY FRECUENCIA

PORCENTAJE

$\begin{array}{llrr} & \text { A } & 56 & 32.18 \\ \text { Derecho } & \text { B } & 24 & 13.79 \\ & \text { C } & 15 & 8.62 \\ \text { Total derecho } & & 95 & 54.60 \\ & \text { A } & 46 & 26.44 \\ \text { Izquierdo } & \text { B } & 20 & 11.49 \\ & \text { C } & 13 & 7.47 \\ \text { Total izquierdo } & & 79 & 45.40 \\ \text { Total } & & 174 & 100.00\end{array}$

TABLA 2. DISTRIBUCIÓN PORCENTUAL DE UNIVERSO Y MUESTRA DE LAS POSICIONES MÁS FRECUENTES CON RESPECTO AL BORDE ANTERIOR DE LA RAMA ASCENDENTE MANDIBULAR SEGÚN PELL Y GREGORY EN PACIENTES QUE FUERON ATENDIDOS POR EXTRACCIÓN DE TERCEROS MOLARES INFERIORES RETENIDOS. FUENTE: PACIENTES DE LA CLÍNICA DE ODONTOLOGÍA - UNIVERSIDAD CATÓLICA DE SANTIAGO DE GUAYAQUIL.

\begin{tabular}{lcrr}
\hline \multicolumn{2}{l}{$\begin{array}{l}\text { PROFUNDIDAD RELATIVA } \\
\text { SEGÚN PELL Y GREGORY }\end{array}$} & FRECUENCIA & PORCENTAJE \\
\hline & I & 22 & 12.64 \\
Derecho & II & 52 & 29.89 \\
& III & 21 & 12.07 \\
Total derecho & & 95 & 54.60 \\
& I & 23 & 13.22 \\
Izquierdo & II & 35 & 20.11 \\
& III & 21 & 12.07 \\
Total izquierdo & & 79 & 45.40 \\
Total & & 174 & 100.00
\end{tabular}

DISCUSIÓN

En la publicación de Susarla et al. (2004) y el de Chaparro- Avendaño et al. (2005) se demostró que son las mujeres quienes acuden más a las consultas odontológicas para procedimiento de extracción de terceros molares, como también se concluyó que la edad más usual esta entre los 18 y 25 años; la muestra de estudio dio resultados similares demostrando que el sexo femenino fue mayor que el de los hombres y que la edad con mayor predisposición fue de 21 a 25 años. ${ }^{20,21,22}$.

En el estudio de Quek et al. (2003) determinaron que la posición según Winter fue la mesoangulada y en la de Pell y Gregory AI con morfología birradiculares. El presente estudio evidenció que la posición según Winter fue también la mesoangulada, ${ }^{21,26}$ en cambio en las clasificaciones de Pell y Gregory nos dio como resultado Tipo II derecho (29,89\%), Tipo II izquierdo (20,11\%), encontrando similitud en la misma clase A. ${ }^{22,23}$

\section{CONCLUSIONES}

De acuerdo a la investigación realizada se recomienda, desarrollar más investigaciones sobre las características radiográficas de los terceros molares inferiores retenidos de los pacientes que acuden a la Clínica de Cirugía de la Universidad Católica de Santiago de Guayaquil, haciendo énfasis en la importancia radiográfica para así evitar complicaciones a futuro y al mismo tiempo estar en la capacidad para afrontarlas.

\section{REFERENCIAS BIBLIOGRÁFICAS}

1. Mateos I, Hernández F. Prevalencia de inclusión dental y patología asociada en pacientes de la Clínica de la Facultad de Odontología Mexicali de la UABC. Revista Odontológica Mexicana. 2005; 84-91. [Accesado 2014 Noviembre 18]. 9(2) Disponible en: http://www. 
journals.unam.mx/index.php/rom/article/ view/16022

2. Cortell I, Silvestre F. Factores predictivos de la dificultad en la extracción del tercer molar incluido: Revisión de la literatura (Primera Parte). Oris. 2006; 34-41. [Accesado 2014 Noviembre 18]. Disponible en: http://www.icoev. es/oris/61-1/articulo2.pdf

3. Cortell I, Silvestre F. Factores predictivos de la dificultad en la extracción del tercer molar incluido: Revisión de la literatura (Segunda Parte). Oris. 2006; 33-41. [Accesado 2014 Noviembre 18]. Disponible en: http://www.icoev. es/oris/62-1/factores_predictivos.pdf

4. Virgili AM, Berini L, Gay Escoda C. Criterios de extracción de los terceros molares incluidos. Anales de Odontología. 2001; 27-35. [Accesado 2014 Noviembre 18]. 1(96) Disponible en: http:// diposit.ub.edu/dspace/handle/2445/25943.

5. Rodríguez G, Martínez E, Duque F, Lodoño L. Caracterización de terceros molares sometidos a exodoncia quirúrgica en la Facultad de Odontología de la Universidad de Antioquia entre 1991 y 2001. Revista de la Facultad de Odontología Universidad de Antioquia. 2007; 76-83. [Accesado 2014 Noviembre 18]. 18(2) Disponible en: http://guajiros.udea.edu.co/ fnsp/cvsp/epidemiologia/Caracterizacion $\% 20 t$ HYPERLINK "http://guajiros.udea.edu.co/fnsp/ cvsp/epidemiologia/Caracterizacion\%20terceros\%20molares....pdf"erceros\%20molares.... HYPERLINK "http:/guajiros.udea.edu.co/ fnsp/cvsp/epidemiologia/Caracterizacion $\% 20$ terceros\%20molares....pdf"pdf

6. Olate S, Alister J, Alveal R, Thomas D, Soto M, Mancilla P, Ceballos M. Hallazgos Clínicos y radiográficos de Terceros Molares con indicación de extracción. Resultaos Preliminares. Int. J. Odontostomat. 2007: 29-34. [Accesado 2014 Noviembre 18]. 1(1) Disponible en: http:// www.scielo.cl/scielo.php?pid =S071795022009000300016\&script=sci_arttext

7. García F, Toro O, Vega M, Verdejo M. Erupción y retención del tercer molar en jóvenes entre 17 y 20 años, Antofagasta, Chile. Int. J. Morphol. 2009: 727-736. [Accesado 2014 Noviembre 18 ]. 27(3) Disponible en: http://www.scielo.cl/scielo.php?pid=S071795022009000300016\&script=sci_arttext

8. Días E, Lima J, Barbosa J, Barreto I, Barbosa L. Prevalencia de la posición de terceros molares inferiores retenidos con la relación a la clasificación de Pell y Gregory. Revista Odontológica
Mexicana. 2009: 229-233. [Accesado $2014 \mathrm{No}^{-}$ viembre 18 ]. 13(4) Disponible en: http://www. medigraphic.com/pdfs/odon/uo- 2009/uo094f. pdf

9. Fuentes R, Borie E, Bustos L, Thomas D. Morfometria de terceros molares: un Estudio de 55 Casos. Int.J.Morphol. 2009: 1285-1289. [Accesado 2014 Noviembre 18 ]. 27(4) Disponible en: http://www. scielo.cl/scielo.php?script=sci_arttext HYPERLINK "http://www.scielo.cl/scielo. php?script=sci_arttext\&amp;pid=S0717-"\& HYPERLINK "http://www.scielo.cl/scielo. php?script=sci_arttext\&amp; pid=S0717"pid=S0717-95022009000400050.

10. Stafne E, Gibilisco J. Oral roentgenographic diagnosis.4a․ ed. Philadelphia: WB Saunders Company; 1975. p. 28 -31. [Accesado 2014 Diciembre 20]. Disponible en: http:// d3i9z8pweb31hm.cloudfront.net/2emalr_oralroentgenographic- diagnosis.pdf

11. Donado M. Cirugía bucal: Patología y técnica 3era edición. Editorial Elsevier España. Año 2005; Capitulo 20: pag. 385.

12. Raspall G. Cirugía Oral e Implantología. Editorial Médica Panamericana. Año 2006; Capítulo 5: pág. 95-124.

13. Morales N. Revista Cubana de Estomatología. Ameloblastoma: Literature Review. pág. 4861. [Accesado 2014 Diciembre 20]. 46(3)

Disponible en: http://www.bvs.sld.cu/revistas/est/vol46_3_09/est06309.pdf

14. Okazaki, K. Journal of Oral Science. Relationship between initial crowding and interproximal forcé during retention phase. Año 2010: pág. 197-201. [Accesado 2014 Diciembre 20]. 52(2) Disponible en: http://www.hindawi. com/journals/tswj/2014/615429/

15. Valdez Berribetia I, Velasco Vivancos V, Berini Aytes L. Revista Europea de Odontoestomatologia; Patomimia Morsicatio Buccarum Et Labiorum. Año 2010; 10:22:26. [Accesado 2014 Diciembre 20]. 10(22) Disponible en: http:// www.redoe.com/ver.php?id=110 panamericana; 2002.

16. Herrera-Atoche J, Colomé-Ruiz G, EscoffiéRamírez M. Agenesia de Terceros Molares, Prevalencia, Distribución y Asociación con otras Anomalías Dentales. Int J Morphol. 2013:1371-1375. [Accesado 2014 Diciembre 20]. 31(4) Disponible en: http://www.scielo. $\mathrm{cl} /$ scielo.php?script=sci_arttext HYPERLINK "http://www.scielo.cl/scielo.php?script=sci_ar ttext\&amp;pid=S0717-95022013000400035"\& 
HYPERLINK "http://www.scielo.cl/ scielo.php?script=sci_arttext\&amp;pid =S0717-95022013000400035"

17. Bachmann H, Cáceres R, Muñoz C, Uribe S. Complicaciones en Cirugía de Terceros Molares entre los Años 2007-2010, en un Hospital Urbano, Chile. Int J Odontostomat. 2014:107-112. [Accesado 2014 Diciembre 20]. 8(1) Disponible en: http://www.scielo.cl/ scielo.php?pid=S0718- 381X2014000100014 HYPERLINK "http://www.scielo.cl/scielo. php?pid=S0718-381X2014000100014\&amp ;script=sci_arttext"\& HYPERLINK "http:// www.scielo.cl/scielo.php?pid=S0718-381X20 14000100014\&amp;script=sci_arttext"script= HYPERLINK "http://www.scielo.cl/scielo. php?pid=S0718-381X2014000100014\&amp;scr ipt=sci_arttext"sci_arttext

18. Beltrán Varas V, Fuentes Fernández R, Bustos Cortés A, Sanhueza Campos A. Relación entre Niveles de Inclusión de Terceros Molares Mandibulares e Índices Antropométricos Físicos de la Mandíbula en Etnias Atacameñas y Aymaras del Norte de Chile. Int J Morphol. 2011:446-454. [Accesado 2014 Diciembre 20]. 29(2) Disponible en: http://www.scielo.cl/pdf/ ijmorphol/v29n2/art25.pdf

19. Henry C. Impacted Third Molar: A Request. BMJ. 1933:845-845. [Accesado 2014 Diciembre 20]. 2(3800) Disponible en: http://www.forp. usp.br/bdj/bdj16\%282\%29/body/v16n2a15.html

20. Cordero E, Vallejos D, Sadovnik I, Romo L. Study of prevalence of third molar according to Pell and Gregory classification. International Journal of Oral and Maxillofacial Surgery. 2011:1127. [Accesado 2014 Diciembre 20]. 40(10) Disponible en: http://www.researchgate.net/ publication/241098743_Study_of_prevalenc HYPERLINK "http://www.researchgate.net/ publication/241098743_Study_of_prevalence_
of_third_molar_according_to_Pell_and_Gregory_classification"e_of_third_molar_according_to_Pell_and_Gregory_classification

21. Almendros-Marqués N, Berini-Aytés L, GayEscoda C. Evaluation of Intraexaminer and Interexaminer Agreement on Classifying Lower Third Molars According to the Systems of Pell and Gregory and of Winter. Journal of Oral and Maxillofacial Surgery. 2008:893-899. [Accesado 2014 Diciembre 20]. 66(5) Disponible en: http:// www.ncbi.nlm.nih.gov/pubmed/18423277

22. Azcárate-Velázquez F, Bertos-Quilez J, Marmesat-Guerrero F, Núñez- Arcos P, HernándezAlfaro F, Ferrés-Padrós E et al. Fiabilidad del uso de la tomografía computarizada de haz cónico en la localización y medida del conducto mandibular en la planificación de técnicas quirúrgicas en el cuerpo mandibular. Revista Española de Cirugía Oral y Maxilofacial. 2014. [Accesado 2014 Diciembre 20)]. Disponible en: http://www.elsevier.es/es-revista-revista-espanola-cirugia-oral- maxilofacial300-pdf-S1130-0558\%2814\%2900034-3-S100

23. Fuentes Fernández R, Oporto Venegas G. Tercer Molar Ectópico Impactado en Zona Retromolar: Reporte de Caso. Int J Morphol. 2009. [Accesado 2014 Diciembre 20]. 27(1) Disponible en: http://www.scielo.cl/scielo.php?pid=S071795 022009000100006\&script=sci_artt 